Bol. Soc. Bot. México 46:25-27 (1984).

\title{
Nuevos registros de Juncaceae y Liliaceae en el Valle de México
}

\author{
Raquel Galván Villanueva ${ }^{1,2}$
}

\begin{abstract}
RESUMEN. Se citan por primera vez para la flora del Valle de México, los siguientes taxa de las familias Juncaceae y Liliaceae: Juncus arcticus Willd. var. andicola (Hook.) Balslev, J. bufonius L., Asphodelus fistulosus L. y Echeandia durangensis (Greenm.) Cruden.
\end{abstract}

ABSTRACTS. The following taxa of Juncaceae and Liliaceae are cited for the first time from the Valley of Mexico: Juncus articus Willd. var. andicola (Hook.) Balslev, J. bufonius L., Asphodelus fistulosus L. and Echeandia durangensis (Greenm.) Cruden.

En el año de 1980 se inició el estudio de tres familias de monocotiledóneas (Juncaceae, Liliaceae y Amaryllidaceae) dentro del proyecto "Flora Fanerogámica del Valle de México". Se tiene programado incluirlas en el último de los tres volúmenes de que constará la obra (primer volumen ya publicado).

A través de la revisión de estos tres grupos de plantas se han encontrado especies y una variedad de las familias Juncaceae y Liliaceae que no se habían citado como elementos de la flora del Valle de México. Ante esta situación se ha creído conveniente dar a conocer estos taxa.

Además de la colecta de plantas vivas se procedió al estudio de los materiales depositados en los herbarios del Instituto de Biología de la Universidad Nacional Autónoma de México (MEXU), de la Escuela Nacional de Ciencias Biológicas (ENCB), del Colegio de Postgraduados de Chapingo (CHAPA), del Instituto Nacional de Investigaciones Forestales (INIF) y de la Comisión Coordinadora para el Desarrollo Agrícola y Ganadero del Estado de México (CODAGEM).

A menos que se haga otra indicación, los especímenes de plantas que se citan a continuación se encuentran depositados en el herbario de la Escuela Nacional de Ciencias Biológicas.

\section{JUNCACEAE}

Juncus arcticus Willd. var. andicola (Hook.) Balslev.

ESTADO DE MÉXICO. Municipio de Amecameca: Vertiente SE del Ixtaccíhuatl, La

\footnotetext{
${ }^{1}$ Escuela Nacional de Ciencias Biológicas, Instituto Politécnico Nacional, 11410 México, D.F.

2 Becaria del Conacyt.
}

Galván-Villanueva R. 1984. Nuevos registros de Juncaceae y Liliaceae en el Valle de México. Boletín de la Sociedad Botánica de México 46: 25-27. 
Joya, Cañada de Alcalican, alt. 3850 m, 1 XI 1965, J. Rzedowski 21576, 14 I 1968, J. Rzedowski 25416, 3 IV 1980, R. Galván 617, 23 XI 1980, R. Galván 718, 28 XII 1980, R. Galván 730.

Especie hasta la fecha únicamente colectada en la parte SE del Valle. Fuera del área de estudio solamente se conoce para México de las Lagunas de Zempoala, Mor. De acuerdo con Balslev $(1979 ; 1982)$ se distribuye además en Guatemala y de Ecuador y del sur de Colombia al norte de Chile y Bolivia.

J. arcticus var. andicola muestra cierta similitud en cuanto a los caracteres vegetativos con otra especie que también prospera en el Valle de México: J. effusus L.; sin embargo, la separación resulta sencilla atendiendo a los caracteres florales. La población en la Joya de Alcalican parece perpetuarse principalmente a través de la reproducción vegetativa y sólo rara vez, quizás cuando las condiciones ambientales y de las plantas son propicias, presenta floración.

\section{Juncus bufonius L.}

HIDALGO. Municipio de Pachuca: Cañada El Bordo-El Cristo (1 km al NNW de Pachuca), alt. 2650 m, 26 VI 1976, M. Medina C. 1399.

ESTADO DE MÉXICO. Municipio de Iturbide: $1 \mathrm{~km}$ al S de la Presa de Iturbide, alt. 3300 m, 8 VI 1980, R. Galván 624. Municipio de Texcoco: San Nicolás Tlamincas, alt. $2300 \mathrm{~m}$, E. Ventura V. $720.8 \mathrm{~km}$ al E de Coatlinchán, alt. $2660 \mathrm{~m}, 7$ XI 1976, J. Rzedowski 34572. Municipio de Tlalmanalco: Santo Tomás Atzingo, $1 \mathrm{~km}$ al NE del poblado, alt. 2550 m, 20 XI 1981, J. García s/n (CHAPA, ENCB).

DISTRITO FEDERAL. Delegación de Cuajimalpa: Puerto de Las Cruces, alt. 3100 m, 9 X 1977, S. González 998, 9 X 1977, J. Rzedowski 35358, 20 VII 1980, R. Galván 643.

Planta inconspicua, generalmente no sobrepasando los $10 \mathrm{~cm}$ de altura, relativamente escasa en el Valle, siendo en ocasiones abundante en pequeños manchones solamente a nivel local. Se distribuye principalmente en las regiones templadas del mundo (Britton y Brown, 1913; Correll y Johnston, 1979). En Norteamérica, con excepción del extremo Norte, se encuentra bien representada. Se han revisado ejemplares de herbario que avalan su presencia en los estados de Baja California Norte, San Luis Potosí, Querétaro, Veracruz, Puebla, Tlaxcala y en otras localidades del Estado de México, fuera de los límites del Valle. Conzatti (1947) y Balslev (1982) la citan además de los estados de Sonora y Chiapas, respectivamente.

\section{LILIACEAE}

Asphodelus fistulosus L.

ESTADO DE MÉXICO. Municipio de Tepotzotlán: $5 \mathrm{~km}$ al $\mathrm{N}$ de Tepotzotlán, sobre la autopista a Querétaro, alt. $2250 \mathrm{~m}, 20$ III 1978, J. Rzedowski $35583.3 \mathrm{~km}$ al N de Tepotzotlán, autopista México-Querétaro, alt. 2250 m, 4 V 1983, R. Galván 1335.

La penetración de la especie al área de estudio parece ser relativamente reciente, y está ocurriendo por la parte oeste del Valle de México a través de la autopista MéxicoQuerétaro. La especie es originaria de la región mediterránea e introducida en América. Se conoce únicamente del estado de California en los Estados Unidos (Munz, 1974) y de México. En este último país es donde $A$. fistulosus parece haber encontrado las condiciones más propicias para su establecimiento y expansión, como lo evidencia su distri- 
bución más o menos continua en el norte y centro del país. Se comporta fundamentalmente como una planta ruderal y su distribución se ha visto favorecida por el incremento en la construcción de carreteras y caminos, así como por el aumento en la circulación de vehículos. Por otra parte la presencia de rizomas cortos, el desarrollo de raíces fibrosas así como su crecimiento cespitoso favorecen un rápido arraigamiento aun en sitios con escasez de suelo. De acuerdo con los registros de herbario se le encuentra en los estados de Durango, Coahuila, Nuevo León, Tamaulipas, Zacatecas, San Luis Potosí, Guanajuato, Querétaro, México, Tlaxcala, Veracruz y Puebla. De acuerdo con Marroquín (1963) se presenta además en el estado de Jalisco.

\section{Echeandia durangensis (Greenm.) Cruden}

ESTADO DE MÉXICO. Municipio de Naucalpan: Puerto El Guarda, $6 \mathrm{~km}$ al W de San Francisco Chimalpa, alt. 3100 m, 12 IX 1969, R. Torres B. 86.

DISTRITO FEDERAL. Delegación de Cuajimalpa: Puerto del Cerro de Las Cruces, carretera México-Toluca, alt. 3100 m, 29 VII 1976, S.D. Koch 76109. Delegación de Tlalpan: Alrededores de la estación La Cima, Serranía del Ajusco, alt. $3000 \mathrm{~m}$, 11 VII 1981, R. Galván 915, 19 VII 1981, R. Galván 920, 29 VII 1982, R. Galván 1238.

La planta es más bien escasa en el Valle; solamente es conocida de tres localidades. Se caracteriza por sus anteras no fusionadas y los filamentos espaciada y ligeramente escamosos-erizados. Este último carácter se pierde a menudo en los ejemplares de herbario. La especie fue descrita como Anthericum, por Greenman (1903) a partir de materiales procedentes del estado de Durango; recientemente Cruden (1981) la transfirió al género Echeandia. La planta parece tener una distribución restringida a México y existen además registros de herbario procedentes de los estados de Jalisco, Querétaro, Hidalgo y Morelos.

Agradecimientos. Agradezco al Dr. Jerzy Rzedowski R. y a la Biól. Graciela Calderón de Rzedowski (Escuela Nacional de Ciencias Biológicas, IPN) sus valiosas sugerencias y la revisión crítica del manuscrito. También nuestro agradecimiento a las autoridades y al personal de los herbarios consultados por las facilidades brindadas durante el desarrollo de este trabajo.

\section{LITERATURA CITADA}

Balslev, H. 1979. Juncaceae, En: G. Harling y B. Sparre (Eds.), Flora of Ecuador 11:1-45.

1982. A systematic monograph of the neotropical Juncaceae. Ph. D. Thesis. City University of New York. $495 \mathrm{p}$.

1983. New taxa and combinations in neotropical Juncus (Juncaceae). Brittonia 35(3):302-308.

BritTon, N.L. y H.A. Brown. 1913. An Illustrated flora of the northern United States and Canada. 2a. ed. Vol. I. Reimpresión 1970, por Dover Publications, N.Y. 680 p.

Conzatti, C. 1947. Flora taxonómica mexicana. 2a. ed. Sociedad Mexicana de Historia Natural, México, D.F.

Correll, D.S. y M.C. Johnston. 1970. Manual of the vascular plants of Texas. Texas Research Foundation, Renner, Texas. 1881 p.

Cruden, R.W. 1981. New Echeandia (Liliaceae) from Mexico. Sida 9(2):134-146.

Greenman, J.M. 1903. New and otherwise noteworthy angiosperms from Mexico and Central America. Proc. Amer. Acad. Arts. 39:69-120.

Marroquín, J.S. 1963. Nota sobre la distribución geográfica de Asphodelus fistulosus L. en México. Cact. Suc. Méx. 8(3):71-73.

Munz, A.P. 1974. A flora of southern California. University of California Press, Berkeley. 1086 p. 